\title{
Modeling Nonlinear Optical Phenomena in Nanophotonics
}

\author{
Jorge Bravo-Abad, Shanhui Fan, Steven G. Johnson, John D. Joannopoulos, and Marin Soljačić
}

(Invited Paper)

\begin{abstract}
In this paper, we review various numerical methods currently used to model nonlinear optical processes in nanophotonics. Among the different theoretical frameworks that have been used to study nonlinear photonic structures, we particularly focus on the application of both perturbation theory and coupled-mode theory to the analysis of complex nonlinear nanophotonic devices. This description is illustrated on several examples of how these techniques can be used to design photonic-crystal-based nonlinear devices. In addition, in all these examples, we show that the predictions made by the two mentioned techniques are in a good agreement with the numerical results obtained from a nonlinear finite-difference-time-domain approach to these problems.
\end{abstract}

Index Terms-Coupled-mode theory (CMT), nonlinear nanophotonics, perturbation theory.

\section{INTRODUCTION}

$\mathbf{E}$ VER since the first experimental demonstration of second harmonic generation in a quartz crystal [1], nonlinear optical phenomena have drawn a great deal of both fundamental and applied interest [2]; numerous applications of nonlinear optical effects have already been commercialized. Many of these applications exploit different types of frequency conversion processes that can be obtained in suitably designed nonlinear structures [3]-[8], enabling generation of coherent radiation in frequency regimes, where it is difficult or not possible to have such sources. In addition, nonlinear optical materials have been proposed as the basic elements of all-optical signal processing systems. This research direction was particularly active during the 1980s, when optical bistability in nonlinear materials was extensively studied [9]. However, due to the very weak nonlinear properties of most materials, the typical sizes and operating powers of such devices were far from fulfilling the requirements of feasible optical-integrated components.

Manuscript received May 27, 2007. This work was supported in part by the Army Research Office through the Institute for Soldier Nanotechnologies under Contract DAAD-19-02-D0002.

J. Bravo-Abad, J. D. Joannopoulos, and M. Soljačić are with the Research Laboratory of Electronics and the Department of Physics, Massachusetts Institute of Technology, Cambridge, MA 02139 USA.

S. Fan is with the Edward L. Ginzton Laboratory, Stanford University, Stanford, CA 94305 USA.

S. G. Johnson is with the Department of Mathematics and the Center for Materials Science and Engineering, Massachusetts Institute of Technology, Cambridge, MA 02139 USA.

Color versions of one or more of the figures in this paper are available online at http://ieeexplore.ieee.org

Digital Object Identifier 10.1109/JLT.2007.903547
This picture dramatically changed with the emergence of photonic crystals (PhCs) [10], [11]. By creating structures with a periodic modulation of the refractive index, it is possible to tailor the dispersion relation and the corresponding electromagnetic modes propagating inside such systems. This ability led to successful demonstration of novel optical devices whose characteristic dimensions are smaller than the wavelength of light [12]-[14]. Within the context of nonlinear optics, the localization of light and guiding capabilities of $\mathrm{PhCs}$ have opened new opportunities for the enhancement of nonlinear effects [15]-[17]. In particular, as we describe in this paper, nonlinear PhCs that include point and/or line defects can be used to design ultrafast nanophotonic devices that are several orders of magnitude smaller than their conventional optical counterparts, while operating at much lower power levels [17].

From a theoretical standpoint, the interest in nonlinear $\mathrm{PhCs}$ has motivated the development of accurate numerical methods to model nonlinear nanophotonics. Most of these methods are extensions of the corresponding theoretical frameworks used to characterize the response of linear systems, where plenty of experience has been acquired during the last 15 years [12].

The first theoretical studies of light propagation through nonlinear periodic structures can be traced back to the 1970s [18], [19]. Some years after the appearance of these early studies, the interest in the theoretical analysis of nonlinear periodic structures increased in a great extent due to the discovery of soliton solutions for periodic dielectric structures [20]-[26]. All these studies were mainly devoted to the case of 1-D periodic structures. One of the first rigorous analyses of nonlinear phenomena in 2-D PhCs was published in [27]. In that work, a Green's function approach was developed to obtain analytical formulas for the enhancement of a sum frequency generation process in a 2-D nonlinear $\mathrm{PhC}$ at frequencies close to the photonic band edges. Remarkably, in that work, the authors showed that the low value of the group velocity at those frequencies produces a large increase of the corresponding frequency conversion efficiency.

Multiple scattering theory has also been used to model optical bistability of a microcavity embedded inside of a nonlinear $\mathrm{PhC}$ [28]. In addition, it is worth pointing out that Green's function technique has been employed to compute both the enhancement of second harmonic generation in 2-D $\mathrm{PhCs}$ [29] and the transmission of light through 2-D nonlinear $\mathrm{PhC}$ waveguides [30]. Another notable recent contribution to the analysis of nonlinear nanophotonic devices has been the 
extension of transfer-matrix frequency domain methods to the case of nonlinear structures [31].

Importantly, it has also been recently demonstrated that the combination of perturbation theory, coupled-mode theory (CMT), and finite-difference-time-domain (FDTD) methods permits an efficient characterization of many nonlinear PhC-based devices [17]. It can be stated that the FDTD method provides exact results (with no approximation except for the discretization), but it is difficult to obtain a physical insight on the studied problem from these results. On the other hand, CMT can be considered as a very accurate and intuitive approach, whereas perturbation theory is an accurate, fast, and intuitive theoretical framework. In this paper, we focus on the application of this particular set of numerical methods to the modeling of nonlinear nanophotonic devices.

This paper is organized as follows. In Section II, we present the fundamentals of perturbation theory and its application to nonlinear photonic structures. Section III is devoted to the description of CMT and how it can be applied to nonlinear optics. We conclude in Section IV.

\section{Perturbation Theory And Nonlinear Optics}

Perturbation theory is a very powerful and general tool used in many different areas of physics [32], e.g., classical mechanics and quantum mechanics. This theoretical framework basically establishes that if the eigenmodes and eigenvalues of a certain operator $\hat{H}_{0}$ are known, then it is possible to compute both the approximate eigenmodes and eigenvalues of any operator $\hat{H}$ that can be written as $\hat{H}=\hat{H}_{0}+\Delta \hat{H}$, where $\Delta \hat{H}$ is a small alteration of $\hat{H}$ (later in this section, we shall more accurately define the term small in this context). The resulting solutions that are obtained within the perturbation theory framework are expressed as a power expansion in $\Delta \hat{H}$. The extension of perturbation theory to nanophotonics is rather straightforward, as we shall see in Sections II-A and B. This discussion will be divided in two main parts. First, we will consider how perturbation theory can be used to analyze linear photonic structures whose refractive index is slightly changed by an external excitation (which could, for example, be electrical, thermal, optical, and mechanical). Second, we will consider the application of perturbation theory to truly nonlinear photonic structures: those in which light signal, through nonlinear self-action, acts on itself. In this case, the description will be clarified by analyzing several nonlinear devices based on $\mathrm{PhC}$ microcavities.

\section{A. Externally Induced Variations of the Dielectric Constant}

First, notice that Maxwell's equations can be cast in the form of an eigenvalue problem for both the magnetic and the electric fields [12]. In particular, if we consider a linear dielectric constant distribution $\varepsilon_{0}(\vec{r})$, the equation that governs the electric field of a particular Bloch eigenmode of interest (which, following the Dirac notation, we denote as $\left|\vec{E}_{0}\right\rangle$ ) can be written as

$$
\nabla \times \nabla \times\left|\vec{E}_{0}\right\rangle=\left(\frac{\omega_{0}}{c}\right)^{2} \varepsilon_{0}(\vec{r})\left|\vec{E}_{0}\right\rangle
$$

where a harmonic time dependence $\exp \left(-i \omega_{0} t\right)$ for the electric field has been assumed.

Now, imagine that by means of some external excitation (which, for instance, can be electrical, thermal, optical, mechanical, etc.), we are able to induce a small change in the dielectric constant distribution $\Delta \varepsilon(\vec{r})$ [i.e., we define a new system whose dielectric constant is $\left.\varepsilon(\vec{r})=\varepsilon_{0}(\vec{r})+\Delta \varepsilon(\vec{r})\right]$. Note that this excitation is simpler than the most general nonlinear interaction, in which $\Delta \varepsilon(\vec{r})$ depends on local light intensity, so light typically experiences self-action; that case will be considered later.

Instead of computing the solutions of (1) from scratch (something that might not be trivial, depending on the kind of perturbation we are introducing), we can use perturbation theory and calculate the eigenvectors and eigenvalues of the new problem from the knowledge of the unperturbed solutions $\left(\left|\vec{E}_{0}\right\rangle\right.$ and $\left.\omega_{0}\right)$. If we assume that the contributions of orders $\left(\Delta \varepsilon / \varepsilon_{0}\right)^{n}$ with $n>1$ can be neglected (this is typically a good approximation for systems where $\Delta \varepsilon / \varepsilon_{0} \leq 0.01$, which is a characteristic for nonlinear applications), we can calculate the correction $\Delta \omega$ to the original eigenvalues $\omega_{0}$ as

$\Delta \omega=-\frac{\omega_{0}}{2} \frac{\left\langle\vec{E}_{0}|\Delta \varepsilon| \vec{E}_{0}\right\rangle}{\left\langle\vec{E}_{0}\left|\varepsilon_{0}\right| \vec{E}_{0}\right\rangle}=-\frac{\omega_{0}}{2} \frac{\int d^{3} r \Delta \varepsilon(\vec{r})\left|\vec{E}_{0}(\vec{r})\right|^{2}}{\int d^{3} r \varepsilon_{0}(\vec{r})\left|\vec{E}_{0}(\vec{r})\right|^{2}}$.

Note that the range of applicability of (2) is very general. It can be applied to calculate $\Delta \omega$ for a mode of a point defect, a guided mode, or a certain eigenstate of the bulk. The generalization of perturbative methods to the case of leaky modes can be found, for instance, in [33] and [34]. In addition, notice that once the electric field $\vec{E}_{0}(\vec{r})$ is calculated (something that might be computationally intensive, depending on the particular problem we are studying), (2) can be used to perform a fast computation of the effect of any small perturbation on the system under analysis.

To illustrate this approach, let us consider the case of a PhC coupled-cavity waveguide [35]-[37] [CCW, which is also called coupled-resonator optical waveguide]. It consists of a line of point defects in an otherwise periodic $\mathrm{PhC}$, with the distance between the defects $\Lambda$ greater than the lattice periodicity of the PhC $a$ [see Fig. 1(a)]. In this structure, each of the point defects acts as a resonant cavity. Light propagation in this system can be seen as a tunneling process between the resonant cavities that are forming the waveguide. This kind of light transport is of interest in nonlinear nanophotonics, since it enables design of waveguide modes with very low group velocity $v_{g}$, yet low dispersion. As we shall describe later, a small value of $v_{g}$ typically leads to a saving factor of $\left(c / v_{g}\right)^{2}$ in the power necessary to operate active devices [38] (or equivalently, for a given power, it leads to the same saving factor in the length of the device).

As mentioned before, in a perturbation theory analysis, we first need to examine the linear properties of the structure under study. As an example, we consider here the case of the 2-D PhC CCW shown in Fig. 1(a). Notice that the values of the group velocity along the slow-light mode can be controlled by 

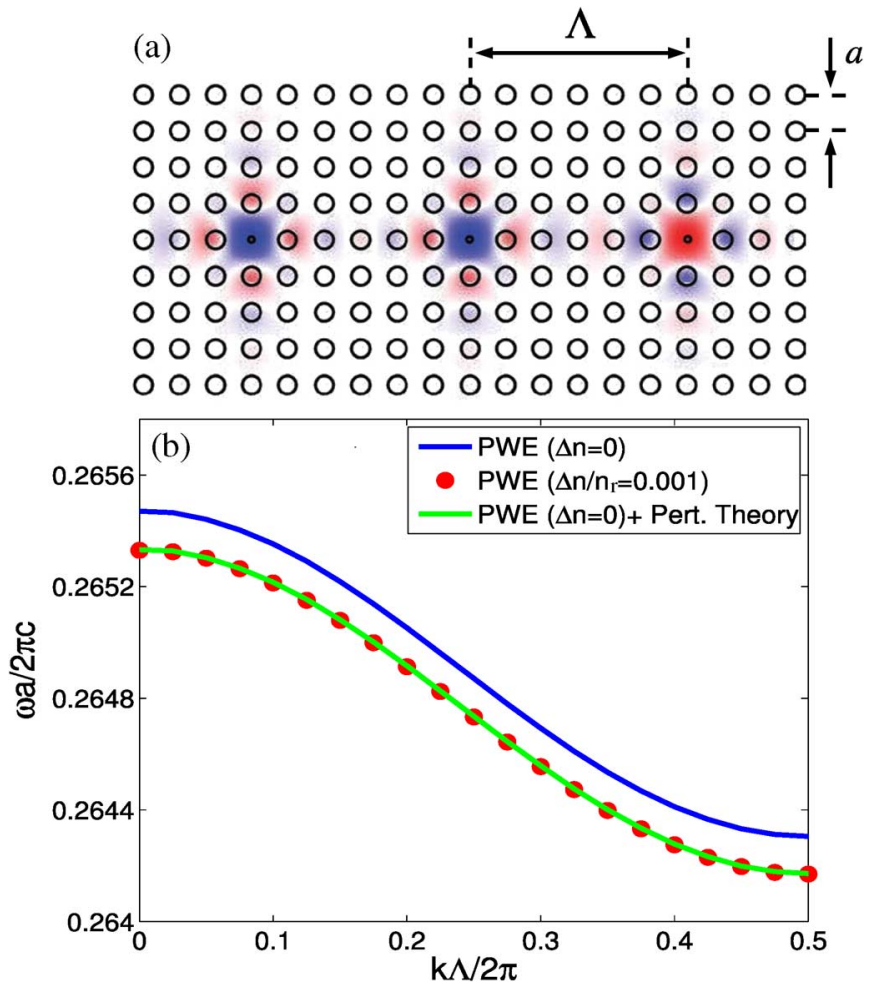

Fig. 1. (a) Electric field distribution of a guided mode of a CCW formed by a 1-D line of defects in a square lattice of high dielectric rods. The highindex rods have a radius $r=0.25 a$, a dielectric constant $\varepsilon_{r}=12.25$, and they are embedded in a material with a lower dielectric constant $\varepsilon_{b}=2.25$. In this case, the defects are created by reducing the radius of the rods from $r$ to $r / 3$ every six lattice periods $\Lambda=6 a$. Red/blue regions correspond to maximum positive/maximum negative amplitude of the electric field, which points in the direction parallel to the rods. (b) Dispersion relation of the $\mathrm{CCW}$ depicted in panel (a). Blue line displays the dispersion relation corresponding to the case without externally induced excitation $\Delta n=0$, which is calculated using the PWE method (see [40]). Reds dots were obtained with a similar calculation but now include an externally induced shift $\Delta n / n_{r}=0.001$ in the refractive index. Green line shows the results obtained by applying perturbation theory to the original case.

changing $\Lambda$ : as we increase the distance between defects $\Lambda$, the value of $v_{g}$ becomes smaller. In addition, it is worth pointing out that the general conclusions for the 2-D case presented here should be valid for CCWs created by introducing defects in 3-D PhCs [39]. Blue line in Fig. 1(b) shows the dispersion relation corresponding to the PhC-based $\mathrm{CCW}$ we are considering. This dispersion relation has been computed by using a frequencydomain method based on a plane wave expansion (PWE) of the fields [40]. For simplicity in Fig. 1(b), we show only the frequencies of the guided modes of the $\mathrm{CCW}$ inside the corresponding photonic bandgap (PBG). As can be observed in Fig. 1(a), the presence of a line of defects creates a guided mode inside the PBG; its electric field is confined in the regions near the point defects.

Let us now consider how the dispersion relation changes if we assume that high-index regions experience a small index change due to some external excitation. For definiteness, we take $\Delta n / n_{r}=0.001$ (see the definition of $n_{r}$ in Fig. 1), which is a typical order of magnitude change for electrooptical or Kerr mechanism. This small value of $\Delta n / n_{r}$ allows us to expect that perturbation theory should produce accurate predictions for the considered system.
Taking into account that $\Delta n / n_{r}$ does not depend on $\vec{r}$ within high- $\varepsilon$ regions and using the first-order expansion $\Delta \varepsilon \approx$ $\varepsilon_{0} 2 \Delta n / n_{r}$, we can rewrite (2) as follows:

$$
\frac{\Delta \omega}{\omega_{0}}=-\frac{\Delta n}{n_{r}} \frac{\int_{\mathrm{HIGH}-\varepsilon} d^{3} r \varepsilon_{0}(\vec{r})\left|\vec{E}_{0}(\vec{r})\right|^{2}}{\int_{\mathrm{SPACE}} d^{3} r \varepsilon_{0}(\vec{r})\left|\vec{E}_{0}(\vec{r})\right|^{2}}
$$

where the volume of integration in the integral in the numerator corresponds only to those regions where $\Delta n$ is applied, i.e., high- $\varepsilon$ regions.

Green line in Fig. 1(b) shows the dispersion relation obtained by applying the shift calculated from (3) to the original dispersion relation (blue line in that figure). The red dots in Fig. 1(b) represent the numerical results directly computed with the mentioned PWE method, assuming $\Delta n / n_{r}=0.001$. As can be seen, for the considered value of $\Delta n$, the exact results and those obtained from perturbation theory are in an excellent agreement.

From the computed dispersion relations displayed in Fig. 1(b), for both the system with and without the external excitation, we can deduce the improvements in the performance introduced by low group velocity waveguides as follows. Many of the active interferometric devices (e.g., Mach-Zehnder interferometers) that could be used in a future integrated optics circuit can be improved by increasing (for a given frequency $\omega)$ the change in the wave vector $k$ of the light propagating inside them. Such devices operate based on the induced phase change $\Delta \phi=L \Delta k$, where $L$ is the length of the device; thus, larger $\Delta k$ is desired, since it leads to a larger $\Delta \phi$ for a given device length. If for small enough values of $\Delta \omega$ we approximate $v_{g} \approx \Delta \omega / \Delta k,(3)$ can be rewritten as

$$
\Delta k=-\frac{\omega_{0}}{v_{g}} \frac{\Delta n}{n_{r}} \frac{\int_{\mathrm{HIGH}-\varepsilon} d^{3} r \varepsilon_{0}(\vec{r})\left|\vec{E}_{0}(\vec{r})\right|^{2}}{\int_{\mathrm{SPACE}} d^{3} r \varepsilon_{0}(\vec{r})\left|\vec{E}_{0}(\vec{r})\right|^{2}} .
$$

Therefore, from (4) together with $\Delta \phi=L \Delta k$, one can see that slow $-v_{g}$ devices present a saving in device length of $c / v_{g}$. In addition, there is typically another saving factor $\sim c / v_{g}$ in the power needed to operate the device coming from the fact that the smaller length of the device means that $\Delta n$ has to be induced in a smaller volume. These savings can often be traded for each other; for example, keeping the length of the device fixed, one can operate it with $\left(c / v_{g}\right)^{2}$ less power, and vice versa.

\section{B. Perturbation Theory and Nonlinear Photonic Structures}

Let us now consider the application of perturbation theory to a truly nonlinear photonic structure, in which light experiences nonlinear self-action. From Maxwell's equations, we can write the following general expression:

$$
\nabla \times[\nabla \times \vec{E}(\vec{r}, t)]=-\frac{1}{c^{2}} \frac{\partial^{2} \vec{D}(\vec{r}, t)}{\partial t^{2}} .
$$

Let us focus on instantaneous Kerr nonlinearity. In that case, if we assume an isotropic nonlinear medium defined by the 
nonlinear susceptibility $\chi^{(3)}$, the displacement electric vector $\vec{D}(\vec{r}, t)$ is of the form

$$
\vec{D}(\vec{r}, t)=\varepsilon_{0}(\vec{r}) \vec{E}(\vec{r}, t)+\chi^{(3)}[\vec{E}(\vec{r}, t)]^{2} \vec{E}(\vec{r}, t) .
$$

By introducing (6) on the right-hand side of (5), we find that

$$
\nabla \times[\nabla \times \vec{E}(\vec{r})]=\frac{\omega^{2} \varepsilon_{0}(\vec{r})}{c^{2}} \vec{E}(\vec{r})+\frac{\omega^{2}}{c^{2}} \vec{P}_{\mathrm{NL}}(\vec{r})
$$

where we have assumed an electric field vector given by

$$
\vec{E}(\vec{r}, t)=\frac{\left[\vec{E}(\vec{r}) \exp (i \omega t)+\vec{E}^{*}(\vec{r}) \exp (-i \omega t)\right]}{2} .
$$

In addition, in (7), we have defined each component of vector $\vec{P}_{\mathrm{NL}}(\vec{r})$ as $P_{\mathrm{NL}, i}=\chi^{(3)} \sum_{j} \xi_{i j} E_{j}$, where $\xi_{i j}=\left[\left(\vec{E} \cdot \vec{E}^{*}\right) \delta_{i j}+\right.$ $\left.\left(E_{i} E_{j}^{*}+E_{i}^{*} E_{j}\right)\right] / 4, i=x, y, z$. We are currently interested in nonlinear self-action only, so we are neglecting interactions with other frequencies: in (7), we have only retained the terms proportional to $\exp ( \pm i \omega t)$.

The quantity $\omega^{2} / c^{2} \vec{P}_{\mathrm{NL}}(\vec{r})$ that appears in the right-hand side of (7) can be treated as a small perturbation [i.e., we assume $\omega=\omega_{0}+\Delta \omega$, where $\omega_{0}$ satisfies (1)]. That approach yields (9), shown at the bottom of the page.

Now, if consider that [2] $n_{2}(\vec{r})=3 \chi^{(3)} /\left(4 n_{0}^{2}(\vec{r})\right)$, where $n_{2}(\vec{r})$ is the local nonlinear Kerr coefficient, (9) can be expressed in (10), shown at the bottom of the page.

Here, it is important to emphasize that the aforementioned equation has been obtained for a particular mode of the system (e.g., for a given $\vec{k}$ ). An analogous perturbation theory can be developed to compute $\Delta \vec{k}$ in optical fibers [41].

In the following part of this section, we will show how (10) can be applied for modeling of realistic nonlinear photonic devices based on PhC microcavities. The linear properties of such system have been extensively analyzed in both 2-D and 3-D PhCs, due to their potential as basic ingredients of compact passive elements of photonic circuits. This research effort has led to the successful demonstration of waveguides [42], sharp bends [43], and channel drop filters [44], whose characteristic dimensions are smaller than the wavelength of light. Recently, these studies have been extended to the case of resonant cavities consisting of a point defect inside a nonlinear PhC [28], [30], [45], [46], showing that these structures can be designed to display optical bistability of the transmitted power with respect to the incident power.

For definiteness, let us consider the 2-D structure depicted in Fig. 2(a), and assume only continuous wave (CW) excitations of the system. It consists of a resonant cavity coupled by a light tunneling mechanism to two single-mode $\mathrm{PhC}$ waveguides placed to the left and to the right of the cavity. In the linear regime, the ratio between the power of the light signal sent through the input waveguide $P_{\mathrm{IN}}$ to the outgoing power through the output waveguide $P_{\text {OUT }}$ has a Lorentzian shape as a function of the input signal frequency $\omega_{0}$, as shown in Fig. 2(b). This transmission function is characterized by a certain frequency width $\Gamma$ and peaks at 1 (i.e., $100 \%$ of the input power is transmitted through the system) whenever $\omega_{0}$ coincides with the resonant frequency of the considered cavity $\omega_{c}$, i.e., $P_{\text {OUT }} / P_{\mathrm{IN}}=\Gamma^{2} /\left(\Gamma^{2}+\left(\omega_{0}-\omega_{c}\right)^{2}\right)$. Imagine now that the material inside the cavity is nonlinear; for instance, we assume that the high-index rods display instantaneous Kerr nonlinearity. If we increase the energy stored in the cavity (e.g., by increasing $P_{\mathrm{IN}}$ ), the self-induced change in the refractive index $\Delta n$ will effectively shift the transmission curve to lower frequencies. The shift of the transmission curve $\Delta \omega$ is given by (10), which in turn is proportional to $P_{\mathrm{OUT}}$, since $P_{\mathrm{OUT}}$ is proportional to the energy stored in the cavity. In fact, one can show [45] that $\Delta \omega$ can be written as

$$
\frac{\Delta \omega}{\omega_{c}}=-\frac{1}{2 c} \kappa Q\left(\left.n_{2}(\vec{r})\right|_{\max }\right) P_{\mathrm{OUT}}
$$

where $\left.n_{2}(\vec{r})\right|_{\max }$ is the maximum value of $n_{2}(\vec{r})$ anywhere, $Q$ is the quality factor of the cavity (i.e., $Q=\omega_{c} / 2 \Gamma$ ), and the parameter $\kappa$ is defined as

$$
\kappa=\left(\frac{c}{\omega_{c}}\right)^{2} \frac{\int_{A} d \vec{r}\left[\left|\vec{E}_{0}(\vec{r}) \vec{E}_{0}(\vec{r})\right|^{2}+2\left|\vec{E}_{0}(\vec{r}) \vec{E}_{0}^{*}(\vec{r})\right|^{2}\right] \frac{n_{0}^{2}(\vec{r})}{3} n_{2}(\vec{r})}{\left.\left[\frac{1}{2} \int_{A} d \vec{r}\left|\vec{E}_{0}(\vec{r})\right|^{2} n_{0}^{2}(\vec{r})\right]^{2} n_{2}(\vec{r})\right|_{\max }}
$$

$$
\frac{\Delta \omega}{\omega_{0}}=-\frac{1}{4} \frac{\int_{\text {ALL VOL. }} d^{3} r \chi^{(3)}\left\{\left[\vec{E}_{0}(r) \cdot \vec{E}_{0}(r)\right]\left[\vec{E}_{0}^{*}(\vec{r}) \cdot \vec{E}_{0}^{*}(\vec{r})\right]+2\left|\vec{E}_{0}(\vec{r})\right|^{4}\right\}}{\int_{\text {ALL VOL. }} d \vec{r}\left|\vec{E}_{0}(\vec{r})\right|^{2} \varepsilon_{0}(\vec{r})}
$$

$$
\frac{\Delta \omega}{\omega_{0}}=-\frac{1}{3} \frac{\int_{\text {ALL VOL. }} d^{3} r n_{0}^{2}(\vec{r}) n_{2}(\vec{r})\left\{\left[\vec{E}_{0}(\vec{r}) \cdot \vec{E}_{0}(\vec{r})\right]\left[\vec{E}_{0}^{*}(\vec{r}) \cdot \vec{E}_{0}^{*}(\vec{r})\right]+2\left|\vec{E}_{0}(\vec{r})\right|^{4}\right\}}{\int_{\text {ALL VOL. }} d \vec{r}\left|\vec{E}_{0}(\vec{r})\right|^{2} n_{0}^{2}(\vec{r})}
$$


(a)
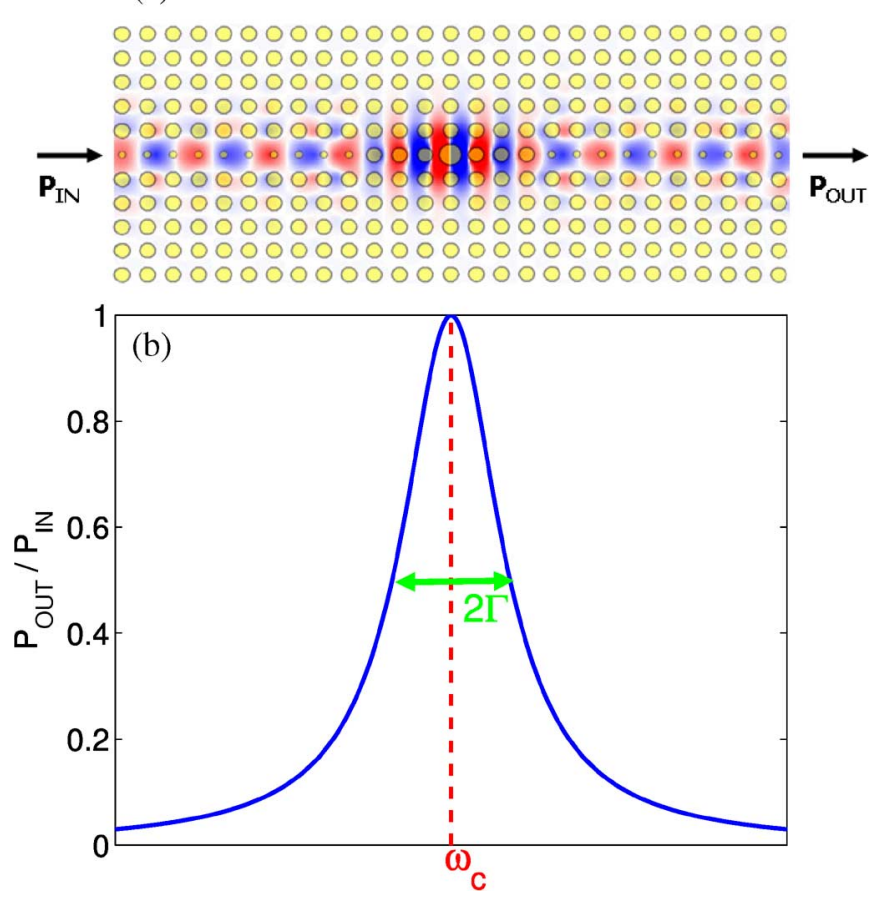

Fig. 2. (a) Distribution of the electric field parallel to the rods of a 2-D PhC microcavity coupled to the left and to the right to two single-mode line-defect waveguides. The structure is formed by a square lattice of high-index rods ( $n_{r}=3.5$, represented as yellow areas) that are embedded in a low dielectric constant material $n_{b}=1.5$. The periodicity of the lattice is given by $a$, whereas the radius of the holes is $r=a / 4$. The resonant cavity has been created in this system by increasing the radius of the central hole to $r_{d}=5 a / 3$. The two line defects have been introduced by reducing the radius of the corresponding holes to $r / 3$. Notice that these line defects act as waveguides that allow the coupling between the microcavity and the external illumination by a tunneling mechanism. (b) Ratio between the outgoing and incoming powers in the linear regime ( $P_{\mathrm{OUT}}$ and $P_{\mathrm{IN}}$, respectively) as a function of the input signal frequency. Vertical dashed line represents the position of resonant frequency of the structure (labeled as $\omega_{c}$ ).

where we have used the same definitions as in (10). $\kappa$ is a measure of the geometric nonlinear feedback efficiency; thus, it is called nonlinear feedback parameter. As can be seen from (12), $\kappa$ is basically the inverse of the modal volume of the particular resonant mode we are studying, weighted by the local Kerr coefficient. In addition, it is important to note that $\kappa$ is almost independent of $n_{2}, Q$, and the peak amplitude of the electric field; thus, a single linear simulation is enough to determine its value for different configurations of the studied system.

From the aforementioned discussion, it follows that the transmission in this nonlinear system depends on $P_{\text {OUT }}$ as $P_{\text {OUT }} / P_{\text {IN }}=\Gamma^{2} /\left(\Gamma^{2}+\left(\omega_{0}-\left(\omega_{c}+\Delta \omega\right)\right)^{2}\right)$. Using (11), this can be written as [45]

$$
\frac{P_{\mathrm{OUT}}}{P_{\mathrm{IN}}}=\frac{1}{1+\left(P_{\mathrm{OUT}} / P_{0}-\delta\right)^{2}}
$$

where $\delta=\left(\omega_{c}-\omega_{0}\right) / \Gamma$, and $P_{0}$ is the characteristic power of the system, which is defined as

$$
P_{0}=\frac{c}{\left.\kappa Q^{2} \omega_{c} n_{2}(\vec{r})\right|_{\max }} .
$$

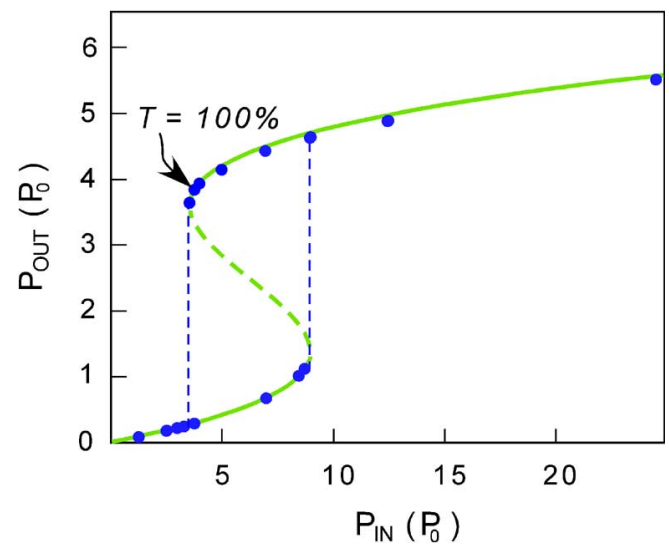

Fig. 3. Output power $P_{\mathrm{OUT}}$ as a function of the input power $P_{\mathrm{IN}}$, corresponding to the structure shown in Fig. 2(a). Green line displays the results obtained from (13) (see text for details about this equation), whereas the blue circles represent the results from FDTD simulations. The dashed line represents the unstable branch of the hysteresis loop. Both perturbation theory and FDTD results numerically confirm the optical bistability displayed by the system shown in Fig. 2(a).

For a given value of the incident power $P_{\mathrm{IN}}$, (13) admits three real solutions for $P_{\text {OUT }}$ whenever $\delta>\sqrt{3}$. This corresponds to the bistability regime of the $\mathrm{PhC}$ microcavity we are considering. Green line in Fig. 3 shows the numerical results for $P_{\text {OUT }} / P_{\text {IN }}$ computed from (13), assuming $\omega_{c}-\omega_{0}=3.8 \gamma$. As can be clearly seen in this figure, this system displays a hysteresis loop, with the dashed line in that figure being the unstable branch of this loop. The bistability properties of this system are confirmed by nonlinear FDTD calculations (shown as blue circles in Fig. 3), which are in a perfect agreement with the predictions made by perturbation theory. Notice that according to (13), $P_{0}$ sets the power level at which one has to operate the device in order to observe bistability. $P_{0}$ scales proportional to $1 /\left(\kappa Q^{2}\right)$, so if we take into account that $\kappa$ roughly scales as $1 / V_{\mathrm{MODE}}$, where $V_{\mathrm{MODE}}$ is the modal volume of the corresponding cavity mode [47], then $P_{0} \propto V_{\mathrm{MODE}} / Q^{2}$. Since in $\mathrm{PhC}$ cavities $V_{\mathrm{MODE}}$ can be very small, whereas $Q$ can be very large, $\mathrm{PhC}$ microcavities are optimal microscopic structures for low power optical bistability. Recently, this kind of bistability properties have been experimentally demonstrated in high- $Q$ $\mathrm{PhC}$ cavities [48], [49]. More examples of the application of perturbation theory for modeling nonlinear $\mathrm{PhC}$ devices can be found in [50] and [51].

\section{CMT in NONLINEAR Optics}

CMT is a fairly general approach valid for a quantitative characterization of systems involving a resonant object weakly coupled to the external environment [52]. It has been successfully applied in a broad range of different fields ranging from photonics to coupled mechanical resonators. In this section, we show how CMT applies to nonlinear nanophotonics. Unlike previous approaches, where only $\mathrm{CW}$ signals can be considered, the CMT framework presented in this section can be used to study signals with arbitrary time dependence. To illustrate the basis of this framework, consider first the example of application of CMT to the 2-D PhC system shown in Fig. 4(a) and (b), which was analyzed in [53]. It is formed in a square array of 
(a)

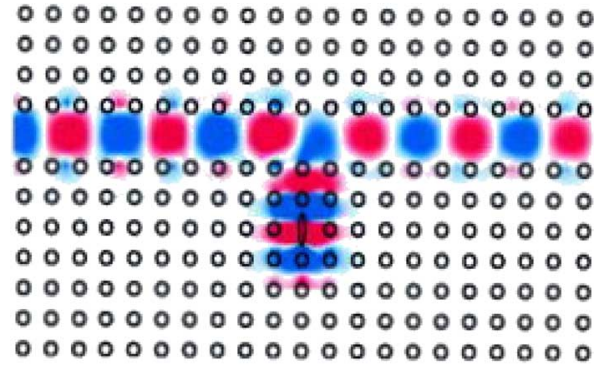

(b)

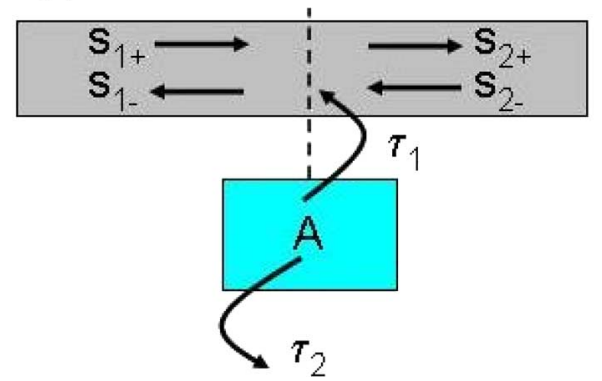

Fig. 4. (a) Distribution of the electric field pointing along the rods in a structure composed by a resonant cavity side-coupled to a line-defect $\mathrm{PhC}$ waveguide. The $\mathrm{PhC}$ is formed by a square array of high-dielectric rods defined by a radius and a refractive index of $0.2 a$ and 3.5 , respectively, where $a$ is the periodicity of the 2-D array. The low-index background is assumed to be air $(n=1)$. The resonant cavity has been created by introducing an elliptical rod, with the length of the long axis being $a$, whereas its short axis length is $0.2 a$. (b) Schematic illustration of the CMT description of the structure shown in panel (a). In this sketch, the meaning of the different quantities appearing in CMT equations is also illustrated.

high dielectric rods $(n=3.5)$ of radius $r=0.2 a$ embedded in air. As can be seen in Fig. 4(a), a waveguide has been introduced in the system by removing a whole row of cylinders. In addition, a resonant cavity has been created by introducing a point defect. In this case, the defect consists of an elliptical dielectric rod, with the length of the long and short axis being $a$ and $0.2 a$, respectively. In the linear case, if we assume that the EM fields inside the cavity are proportional to a certain parameter $A$, this system can be modeled within the CMT framework by means of the following set of equations [see the corresponding sketch in Fig. 4(b)]:

$$
\begin{aligned}
\frac{d A}{d t} & =i \omega_{c} A-\frac{1}{\tau_{1}} A-\frac{1}{\tau_{2}} A+\kappa s_{1+}+\kappa s_{2-} \\
s_{1-} & =s_{2-}-\kappa A \\
s_{2+} & =s_{1+}-\kappa A
\end{aligned}
$$

where $\omega_{c}$ is the resonant frequency of the cavity. $\tau_{1}$ is the decay constant of the cavity into the waveguide modes propagating to the right and to the left, whereas the magnitude $\tau_{2}$ governs the decay rate due to intrinsic losses of the cavity (like, for instance, the absorption losses of the corresponding material). $s_{1+}$ and $s_{1-}\left(s_{2+}\right.$ and $\left.s_{2-}\right)$ represent the complex amplitudes of the fields propagating to the left and right at the input (output) of the waveguide. The parameter $\kappa$ governs the input coupling between the resonant cavity and the propagating modes inside the waveguide. From power conservation arguments [52], it can be deduced that this magnitude is given by $\kappa=\sqrt{1 / \tau_{1}}$. Note that the parameter $A$ is normalized so that $|A|^{2}$ is the energy

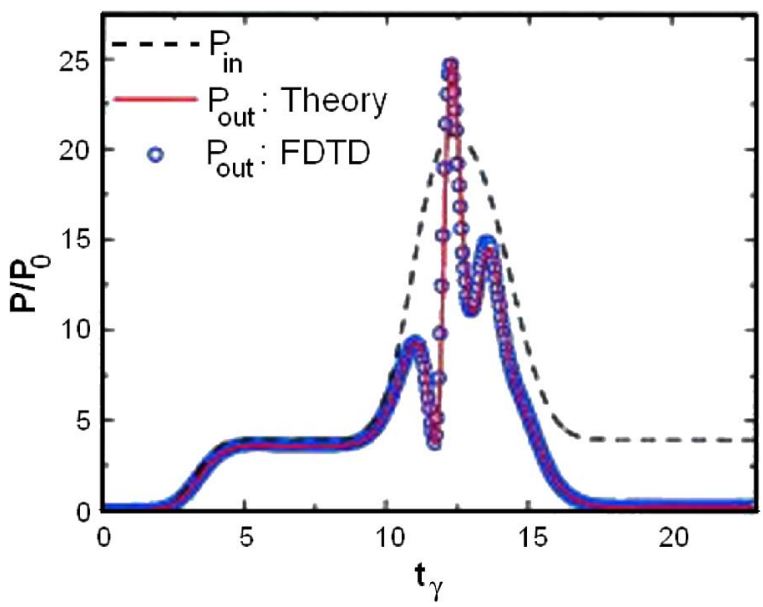

Fig. 5. Temporal dependence of the input and output powers computed for the 2-D PhC structure shown in Fig. 4(a). Parameter $t_{\gamma}$ represents the product $t \gamma$ (see the definition of $\gamma$ in the text). The black dashed line displays the input power, the red line corresponds to the results from CMT, and the blue dots correspond to FDTD results. Notice how, in this case, CMT accurately reproduces the behavior simulated with an FDTD method.

inside the cavity, whereas $s_{1,2 \pm}$ are normalized so that $\left|s_{1,2 \pm}\right|^{2}$ represent the corresponding incoming/outgoing powers inside the waveguides.

Now, if we assume that $s_{2-}=0$ (the input signal launched only from the left) and that external losses can be neglected $\left(\tau_{2} \rightarrow \infty\right),(15)-(17)$ can be expressed in terms of just $s_{1+}$ and $s_{1-}$ as

$$
\frac{d s_{1-}}{d t}=i \omega_{c} s_{1-}-\frac{1}{\tau_{1}} s_{1-}-\frac{1}{\tau_{1}} s_{1+} .
$$

Therefore, once we solve (18), we can compute the output power through the system $P_{\text {OUT }}$ given a certain input power $P_{\mathrm{IN}}$ just by computing $P_{\mathrm{IN}}=\left|s_{1+}\right|^{2}$ and $P_{\mathrm{OUT}}=\left|s_{1+}\right|^{2}-$ $\left|s_{1-}\right|^{2}$.

Next, assume that the region close to the defect has an instantaneous Kerr nonlinearity. In that case, from a perturbation theory analysis, we know that the nonlinearity introduces a frequency shift to the resonant frequency proportional to $P_{\text {OUT }}$ [see (11)]. If we also take into account (14), nonlinear effects can be introduced in (18) just by replacing $\omega_{c}$ by the corresponding shifted frequency that was obtained from a perturbation theory analysis [see (11)]. This procedure yields

$$
\frac{d s_{1-}}{d t}=i \omega_{c}\left(1-\frac{1}{2 Q} \frac{\left|s_{1-}\right|^{2}}{P_{0}}\right) s_{1-}-\frac{1}{\tau_{1}} s_{1-}-\frac{1}{\tau_{1}} s_{1+}
$$

where $Q$ is the quality factor of the cavity (i.e., $Q=\omega_{c} \tau_{1} / 2$ ), and $P_{0}$ is defined in (14).

In order to show the accuracy of this approach, Fig. 5 shows the comparison between the CMT results for the outgoing power as a function of time (red line) and the numerical results obtained from a nonlinear FDTD method (blue circles). In that figure, the temporal dependence of the input power is shown as a black dashed line. Notice how the input signal switch the system from a high transmission state to a low transmission one. For another example of using CMT to model nonlinear $\mathrm{PhC}$ devices, see [54]. 


\section{CONCLUSION}

The rapid advance of large-scale computing technologies is leading to an unprecedented ability to design and simulate novel photonic devices through virtually exact methods such as the FDTD method. In particular, the application of the FDTD method to nonlinear structures has been proven to be an essential technique in the modeling of novel nonlinear nanophotonic devices. However, FDTD simulations can be seen as numerical experiments in which the physical mechanisms responsible for the behavior of the system we are analyzing are often difficult to elucidate.

Thus, in order to get physical insight into the nonlinear optical response of nanophotonic structures, developing approximate analytical methods is crucial; these methods allow us to propose nonlinear devices with novel functionalities.

In this paper, we have reviewed how both perturbation theory and CMT can be used to characterize the behavior of complex nonlinear systems. In particular, we have focused in the application of these methods to nonlinear PhCs, since these structures are one of the most promising candidates for largescale all-optical integration. Different examples that are shown in this paper extensively demonstrate good agreement between perturbation theory, CMT, and FDTD results.

\section{REFERENCES}

[1] P. A. Franken, A. E. Hill, C. W. Peters, and G. Weinreich, "Generation of optical harmonics," Phys. Rev. Lett., vol. 7, no. 4, pp. 118-199, Aug. 1961.

[2] R. W. Boyd, Nonlinear Optics. San Diego, CA: Academic, 1992.

[3] J. A. Armstrong, N. Bloembergen, J. Ducuing, and P. S. Pershan, "Interactions between light waves in a nonlinear dielectric," Phys. Rev., vol. 127, no. 6, pp. 1918-1939, Sep. 1962.

[4] M. M. Fejer, G. A. Magel, H. D. Jundt, and R. L. Byer, "Quasi-phasematched 2nd harmonic-generation-Tuning and tolerances," IEEE J. Quantum Electron., vol. 28, no. 11, pp. 2631-2654, Nov. 1992.

[5] L. E. Myers, G. D. Miller, R. C. Eckardt, M. M. Fejer, R. L. Byer, and W. R. Bosenberg, "Quasi-phase-matched 1.064-mu-m-pumped optical parametric oscillator in bulk periodically poled LiNbO3," Opt. Lett., vol. 20, no. 1, pp. 52-54, Jan. 1995

[6] S. J. B. Yoo, C. Caneau, R. Bhat, M. A. Koza, A. Rajhel, and N. Antoniades, "Wavelength conversion by difference frequency generation in AlGaAs waveguides with periodic domain inversion achieved by wafer bonding," Appl. Phys. Lett., vol. 68, no. 19, pp. 2609-2611, May 1996.

[7] H. Karlsson and F. Laurell, "Electric field poling of flux grown KTiOPO4," Appl. Phys. Lett., vol. 71, no. 24, pp. 3474-3476, Dec. 1997.

[8] V. Berger, "Nonlinear photonic crystals," Phys. Rev. Lett., vol. 81, no. 19, pp. 4136-4139, Nov. 1998.

[9] H. Gibbs, Optical Bistability: Controlling Light With Light. New York: Academic, 1985.

[10] E. Yablonovitch, "Inhibited spontaneous emission in solid-state physics and electronics," Phys. Rev. Lett., vol. 58, no. 20, pp. 2059-2062, May 1987.

[11] S. John, "Strong localization of photons in certain disordered dielectric superlattices," Phys. Rev. Lett., vol. 58, no. 23, pp. 2486-2489, Jun. 1987.

[12] J. D. Joannopoulos, R. D. Meade, and J. N. Winn, Photonic Crystals: Molding the Flow of Light. Princeton, NJ: Princeton Univ., 1995.

[13] K. Sakoda, Optical Properties of Photonic Crystals. Berlin, Germany: Springer-Verlag, 2001.

[14] S. G. Johnson, Photonic Crystals: The Road From Theory to Practice. Norwell, MA: Kluwer, 2002.

[15] C. M. Bowden and A. M. Zheltikov, "Nonlinear optics of photonic crystals," J. Opt. Soc. Amer. B, Opt. Phys., vol. 19, no. 9, pp. 2046-2048, Sep. 2002.

[16] R. E. Slusher and B. J. Eggleton, Nonlinear Photonic Crystals. Berlin, Germany: Springer-Verlag, 2003.
[17] M. Soljačić and J. D. Joannopoulos, "Enhancement of nonlinear effects using photonic crystals," Nat. Mater, vol. 3, no. 4, pp. 211-219, Apr. 2004.

[18] H. Kogelnik and C. V. Shank, "Stimulated emission in a periodic structure," Appl. Phys. Lett., vol. 18, no. 4, pp. 152-154, Feb. 1971.

[19] H. G. Winful, J. H. Marburger, and E. Garmire, "Theory of bistability in non-linear distributed feedback structures," Appl. Phys. Lett., vol. 35, no. 5, pp. 379-381, Sep. 1979.

[20] W. Chen and D. L. Mills, "Gap solitons and the nonlinear opticalresponse of superlattices," Phys. Rev. Lett., vol. 58, no. 2, pp. 160-163, Jan. 1987.

[21] D. L. Mills and S. E. Trullinger, "Gap solitons in nonlinear periodic structures," Phys. Rev. B, Condens. Matter, vol. 36, no. 2, pp. 947-952, Jul. 1987.

[22] J. E. Sipe and H. G. Winful, "Nonlinear Schrodinger solitons in a periodic structure," Opt. Lett., vol. 13, no. 2, pp. 132-133, Feb. 1988.

[23] D. N. Christodoulides and R. I. Joseph, "Slow Bragg solitons in nonlinear periodic structures," Phys. Rev. Lett., vol. 62, no. 15, pp. 1746-1749, Apr. 1989.

[24] A. B. Aceves and S. Wabnitz, "Self-induced transparency solitons in nonlinear refractive periodic media," Phys. Lett. A, vol. 141, no. 1/2, pp. 37-42, Oct. 1989.

[25] C. M. de Sterke and J. E. Sipe, "Extensions and generalizations of an envelope-function approach for the electrodynamics of nonlinear periodic structures," Phys. Rev. A, Gen. Phys., vol. 39, no. 10, pp. 5163-5178, May 1989.

[26] G. D'Aguanno, M. Centini, M. Scalora, C. Sibilia, Y. Dumeige, P. Vidakovic, J. A. Levenson, M. J. Bloemer, C. M. Bowden, J. W. Haus, and M. Bertolotti, "Photonic band edge effects in finite structures and applications to chi(2) interactions," Phys. Rev. E, Stat. Phys. Plasmas Fluids Relat. Interdiscip. Top., vol. 64, no. 1, p. 16609, Jul. 2001.

[27] K. Sakoda and K. Ohtaka, "Sum-frequency generation in a twodimensional photonic lattice," Phys. Rev. B, Condens. Matter, vol. 54 no. 8, pp. 5742-5749, Aug. 1996.

[28] E. Centeno and D. Felbacq, "Optical bistability in finite-size nonlinear bidimensional photonic crystals doped by a microcavity," Phys. Rev. B, Condens. Matter, vol. 62, no. 12, pp. R7683-R7686, Sep. 2000.

[29] A. R. Cowan and J. F. Young, "Mode matching for second-harmonic generation in photonic crystal waveguides," Phys. Rev. B, Condens. Matter, vol. 65, no. 8, p. 085106, Feb. 2002.

[30] S. F. Mingaleev and Y. S. Kivshar, "Nonlinear transmission and light localization in photonic-crystal waveguides," J. Opt. Soc. Amer. A, Opt. Image Sci., vol. 19, no. 9, pp. 2241-2249, Sep. 2002.

[31] B. Maes, P. Bienstman, and R. Baets, "Modeling second-harmonic generation by use of mode expansion," J. Opt. Soc. Amer. B, Opt. Phys., vol. 22, no. 7, pp. 1378-1383, Jul. 2005.

[32] P. M. Morse and H. Feshbach, Methods of Theoretical Physics. New York: McGraw-Hill, 1953.

[33] A. W. Snyder and J. D. Love, Optical Waveguide Theory. London, U.K.: Chapman \& Hall, 1983.

[34] W. Suh, Z. Wang, and S. Fan, "Temporal coupled-mode theory and the presence of non-orthogonal modes in lossless multimode cavities," IEEE J. Quantum Electron., vol. 40, no. 10, pp. 1511-1518, Oct. 2004.

[35] A. Yariv, Y. Xu, R. K. Lee, and A. Scherer, "Coupled-resonator optical waveguide: A proposal and analysis," Opt. Lett., vol. 24, no. 11, pp. 711713, Jun. 1999.

[36] S. Mookherjea and A. Yariv, "Second-harmonic generation with pulses in a coupled-resonator optical waveguide," Phys. Rev. E, Stat. Phys. Plasmas Fluids Relat. Interdiscip. Top., vol. 65, no. 2, p. 026607, Feb. 2002.

[37] M. Bayindir, B. Temelkuran, and E. Ozbay, "Tight-binding description of the coupled defect modes in three-dimensional photonic crystals," Phys. Rev. Lett., vol. 84, no. 10, p. 2140, Mar. 2000.

[38] M. Soljačić, S. G. Johnson, S. Fan, M. Ibanescu, E. Ippen, and J. D. Joannopoulos, "Photonic-crystal slow-light enhancement of nonlinear phase sensitivity," J. Opt. Soc. Amer. B, Opt. Phys., vol. 19, no. 9, pp. 2052-2059, Sep. 2002.

[39] M. L. Povinelli, S. G. Johnson, S. Fan, and J. D. Joannopoulos, "Emulation of two-dimensional photonic crystal defect modes in a photonic crystal with a three-dimensional photonic band gap," Phys. Rev. B, Condens. Matter, vol. 64, no. 7, p. 0753131, Aug. 2001.

[40] S. G. Johnson and J. D. Joannopoulos, "Block-iterative frequency-domain methods for Maxwell's equations in a planewave basis," Opt. Express, vol. 8, no. 3, pp. 173-190, Jan. 2001.

[41] V. P. Tzolov, M. Fontaine, N. Godbout, and S. Lacroix, "Nonlinear selfphase-modulation effects: A vectorial first-order perturbation approach," Opt. Lett., vol. 20, no. 5, pp. 456-459, Mar. 1995. 
[42] S. Noda, K. Tomoda, N. Yamamoto, and A. Chutinan, "Full threedimensional photonic bandgap crystals at near-infrared wavelengths," Science, vol. 289, no. 5479, pp. 604-606, Jul. 2000.

[43] E. Chow, S. Y. Lin, J. R. Wendt, S. G. Johnson, and J. D. Joannopoulos, "Quantitative analysis of bending efficiency in photonic-crystal waveguide bends at lambda $=1.55 \mathrm{mu}$ m wavelengths," Opt. Lett., vol. 26, no. 5, pp. 286-288, Mar. 2001.

[44] S. Fan, P. R. Villenueve, J. D. Joannopoulos, and H. A. Haus, "Channel drop tunneling through localized states," Phys. Rev. Lett., vol. 80, no. 5, pp. $960-963$, Feb. 1998.

[45] M. Soljačić, M. Ibanescu, S. G. Johnson, Y. Fink, and J. D. Joannopoulos, "Optimal bistable switching in nonlinear photonic crystals," Phys. Rev. E, Stat. Phys. Plasmas Fluids Relat. Interdiscip. Top., vol. 66, no. 5, p. 055601R, Nov. 2002.

[46] A. R. Cowan and J. F. Young, "Optical bistability involving photonic crystal microcavities and Fano line shapes," Phys. Rev. E, Stat. Phys. Plasmas Fluids Relat. Interdiscip. Top., vol. 68, no. 4, p. 046606 , Oct. 2003.

[47] Y. Xu, R. K. Lee, and A. Yariv, "Quantum analysis and the classical analysis of spontaneous emission in a microcavity," Phys. Rev. A, Gen. Phys., vol. 61, no. 3, p. 33808 , Mar. 2000.

[48] M. Notomi, A. Shinya, S. Mitsugi, G. Kira, E. Kuramochi, and T. Tanabe, "Optical bistable switching action of Si high-Q photonic-crystal nanocavities," Opt. Express, vol. 13, no. 7, pp. 2678-2687, Apr. 2005.

[49] P. E. Barclay, K. Srinivasan, and O. Painter, "Nonlinear response of silicon photonic crystal microresonators excited via an integrated waveguide and fiber taper," Opt. Express, vol. 13, no. 3, pp. 801-820, Feb. 2005.

[50] M. Soljačić, C. Luo, J. D. Joannopoulos, and S. Fan, "Nonlinear photonic crystal microdevices for optical integration," Opt. Lett., vol. 28, no. 8, pp. 637-639, Apr. 2003.

[51] M. Soljačić, M. Ibanescu, S. G. Johnson, J. D. Joannopoulos, and Y. Fink, "Optical bistability in axially modulated OmniGuide fibers," Opt. Lett., vol. 28, no. 7, pp. 516-518, Apr. 2003.

[52] H. A. Haus, Waves and Fields in Optoelectronics. Englewood Cliffs, NJ: Prentice-Hall, 1984.

[53] M. F. Yanik, S. Fan, and M. Soljačić, "High-contrast all-optical bistable switching in photonic crystal microcavities," Appl. Phys. Lett., vol. 83, no. 14, pp. 2739-2741, Oct. 2003.

[54] M. F. Yanik, S. Fan, M. Soljačić, and J. D. Joannopoulos, "All-optical transistor action with bistable switching in a photonic crystal crosswaveguide geometry," Opt. Lett., vol. 28, no. 24, pp. 2506-2508, Dec. 2003

Jorge Bravo-Abad received the B.S. and Ph.D. degrees in physics from the Universidad Autonoma de Madrid, Madrid, Spain, in 2001 and 2006, respectively.

Since 2007, he has been a Postdoctoral Researcher with the Research Laboratory of Electronics and the Department of Physics, Massachusetts Institute of Technology, Cambridge. His current research interests include nonlinear photonic crystals and plasmonics.

Shanhui Fan received the Ph.D. degree in theoretical condensed matter physics from the Massachusetts Institute of Technology (MIT), Cambridge, in 1997.

$\mathrm{He}$ is an Assistant Professor of electrical engineering with the Edward L. Ginzton Laboratory, Stanford University, Stanford, CA. He was a Research Scientist in the Research Laboratory of Electronics, MIT prior to his appointment at Stanford University. He has published over 130 refereed journal articles and has given over 90 invited talks. He is the holder of 28 U.S. patents. His research interests include computational and theoretical studies of solid state and photonic structures and devices, particularly photonic crystals, microcavities, and nanophotonic circuits and elements.

Dr. Fan is a member of the IEEE Antennas and Propagation Society, Optical Society of America (OSA), and SPIE-The International Society for Optical Engineering. He is the recipient of a National Science Foundation Career Award (2002), a David and Lucile Packard Fellowship in Science and Engineering (2003), the National Academy of Sciences Award for Initiative in Research (2007), and the Adolph Lomb medal for the OSA (2007).
Steven G. Johnson received the Ph.D. degree in condensed matter physics from the Massachusetts Institute of Technology (MIT), Cambridge.

$\mathrm{He}$ is currently an Assistant Professor of applied mathematics with the Department of Mathematics and the Center for Materials Science and Engineering, MIT. He is the author or coauthor of nearly 80 papers on nanophotonics and electromagnetism in media structured on the wavelength scale. His thesis was published as a book entitled Photonic Crystals: The Road from Theory to Practice (Kluwer, 2001). His research interests include the development of semianalytical models of nanophotonic systems, design of devices (such as hollow-core fibers for medical lasers), and high-performance computation (with much of his software available on the Web).

Dr. Johnson is the recipient of the 1999 J. H. Wilkinson Prize for Numerical Software for his work on the FFTW fast Fourier transform library.

John D. Joannopoulos received the B.A. and Ph.D. degrees from the University of California, Berkeley, in 1968 and 1974, respectively.

He was with the Faculty of Physics, Massachusetts Institute of Technology (MIT), Cambridge, as an Assistant Professor during 1974-1978, an Associate Professor during 1978-1983, and a Professor during 1983-1996. Since 1996, he has been with the Research Laboratory of Electronics and the Department of Physics, MIT, as the Francis Wright Davis Chair of Professor of Physics. He has served as a Divisional Associate Editor of Physical Review Letters, a member of Editorial Board of Reviews of Modern Physics, and is currently the Director of the Institute for Soldier Nanotechnologies at MIT. His research has been in theoretical condensed matter physics. He is responsible for the development of numerous calculation methods for the study of elementary excitations in complex many-particle systems. He is the author or coauthor of more than 450 refereed scientific journal articles and two textbooks on photonic crystals. $\mathrm{He}$ is the holder of 45 U.S. patents. His research interests include the electronic, vibrational, and optical structure of crystalline and amorphous bulk solids, their surfaces, interfaces, and defects; localization in disordered systems; phase transitions and critical phenomena; and, over the past decade, the theory of photonic bandgap crystals. Since 2003, he has been one of the Thompson ISI most Highly Cited Researchers.

Prof. Joannopoulos is a Fellow of the American Physical Society (1983) and a Fellow of the American Association for the Advancement of Science (2002). He was an Alfred P. Sloan Fellow (1976-1980) and a John S. Guggenheim Fellow (1981-1982). He is the recipient of the Student Council Graduate Teaching Award (1991), the William Buechner Teaching Prize (1996), the David Adler Award of the American Physical Society (1997), and the School of Science Graduate Teaching Award (2002).

Marin Soljačić received the B.S.E. degree in physics and electrical engineering from the Massachusetts Institute of Technology (MIT), Cambridge, in 1996 and the Ph.D. degree in physics from Princeton University, Princeton, NJ, in 2000.

In September 2000, he was named an MIT Pappalardo Fellow in Physics. In 2003, he was appointed as a Principal Research Scientist in the Research Laboratory of Electronics and the Department of Physics, MIT, where he has been an Assistant Professor of physics since September 2005.

Dr. Soljačić is the recipient of the Adolph Lomb medal from the Optical Society of America (2005) and has been chosen by Technology Review to be one of the "TR35": top 35 innovators under the age of 35 (2006). 